\title{
The Strong Convergence of Prediction-Correction and Relaxed Hybrid Steepest-Descent Method for Variational Inequalities
}

\author{
Haiwen $\mathrm{Xu}^{1,2}$ \\ ${ }^{1}$ School of Computer Science, Civil Aviation Flight University of China, Guanghan 618307, China \\ ${ }^{2}$ School of Civil Aviation, Nanjing University of Aeronautics and Astronautics, Nanjing 210016, China \\ Correspondence should be addressed to Haiwen Xu; xuhaiwen_dream@163.com
}

Received 22 June 2013; Accepted 19 August 2013

Academic Editor: Xu Minghua

Copyright (C) 2013 Haiwen Xu. This is an open access article distributed under the Creative Commons Attribution License, which permits unrestricted use, distribution, and reproduction in any medium, provided the original work is properly cited.

\begin{abstract}
We establish the strong convergence of prediction-correction and relaxed hybrid steepest-descent method (PRH method) for variational inequalities under some suitable conditions that simplify the proof. And it is to be noted that the proof is different from the previous results and also is not similar to the previous results. More importantly, we design a set of practical numerical experiments. The results demonstrate that the PRH method under some descent directions is more slightly efficient than that of the modified and relaxed hybrid steepest-descent method, and the PRH Method under some new conditions is more efficient than that under some old conditions.
\end{abstract}

\section{Introduction}

Let $H$ be a real Hilbert space with inner product $\langle\cdot, \cdot\rangle$ and norm $\|\cdot\|$, let $K$ be a nonempty closed convex subset of $H$, and let $F: H \rightarrow H$ be an operator. Then the variational inequality problem $\mathrm{VI}(F, K)[1]$ is to find $x^{*} \in K$ such that

$$
x^{*} \in K, \quad\left\langle x-x^{*}, F\left(x^{*}\right)\right\rangle \geq 0, \quad \forall x \in K .
$$

The literature contains many methods for solving variational inequality problems; see [2-25] and references therein. According to the relationship between the variational inequality problems and a fixed point problem, we can obtain

$$
\begin{aligned}
& x^{*} \text { is the solution of } \operatorname{VI}(F, K) \\
& \Longleftrightarrow x^{*}=P_{K}\left[x^{*}-\beta F\left(x^{*}\right)\right], \quad \beta>0,
\end{aligned}
$$

where the projection operator $P_{K}$ is the projection from $H$ onto $K$, that is,

$$
P_{K}(x)=\underset{y \in K}{\operatorname{argmin}}\|x-y\|, \quad \forall x \in H .
$$

In this paper, $F: H \rightarrow H$ is an operator with $F: \kappa$-Lipschtz and $\eta$-strongly monotone; that is, $F$ satisfies the following conditions:

$$
\begin{gathered}
\|F(x)-F(y)\| \leq \kappa\|x-y\|, \\
\langle F(x)-F(y), x-y\rangle \geq \eta\|x-y\|^{2}, \quad \forall x, y \in K .
\end{gathered}
$$

If $\beta$ is small enough, then $P_{K}$ is a contraction. Naturally, the convergence of Picard iterates generated by the right-hand side of (2) is obtained by Banach's fixed point theorem. Such a method is called the projection method or more results about the projection method see $[6,8,20]$ and so forth.

In fact, the projection $P_{K}$ in the contraction methods may not be easy to compute, and a great effort is to compute the projection $P_{K}$ in each iteration. Yamada and Deutsch have provided a hybrid steepest-descent method for solving the $\operatorname{VI}(F, K)[2,3]$ in order to reduce the difficulty and complexity of computing the projection $P_{K}$. Subsequently, the convergence of hybrid steepest-descent methods was given out by Xu and Kim [4] and Zeng et al. [5]. Naturally, by analyzing several three-step iterative methods in each iteration by the fixed pointed equation, we can obtain the Noor iterations. Recently, Ding et al. [7] proposed a threestep relaxed hybrid steepest-descent method for variational 
inequalities, and the simple proof of three-step relaxed hybrid steepest-descent methods under different conditions was introduced by Yao et al. [24]. The literature [14, 16] described a modified and relaxed hybrid steepest-descent (MRHSD) method and the different convergence of the MRHSD method under the different conditions. A set of practical numerical experiments in the literature [16] demonstrated that the MRHSD method has different efficiency under different conditions. Subsequently, the prediction-correction and relaxed hybrid steepest-descent method (PRH method) [15] makes more use of the history information and less decreases the loss of information than the methods $[7,14]$. The PRH method introduced more descent directions than the MRHSD method $[14,16]$, and computing these descent directions only needs the history information.

In this paper, we will prove the strong convergence of PRH method under different and suitable restrictions imposed on parameters (Condition 12), which differs from that of [15]. Moreover, the proof of strong convergence is different from the previous proof in [15], which is not similar to that in [7] in Step 2. And more importantly, numerical experiments verify that the $\mathrm{PRH}$ method under Condition 12 is more efficient than that under Condition 10, and the PRH method under some descent directions is more slightly efficient than that of the MRHSD method $[14,16]$. Furthermore, it is easy to obtain these descent directions.

The remainder of the paper is organized as follows. In Section 2, we review several lemmas and preliminaries. We prove the convergence theorem under Condition 12 in Section 3. In Section 4, we give out a series of numerical experiments, which demonstrated that the PRH method under Condition 12 is more efficient than under Condition 10. Section 5 concludes the paper.

\section{Preliminaries}

In order to proof the later convergence theorem, we introduce several lemmas and the main results in the following.

Lemma 1. In a real Hilbert space $H$, there holds the inequality

$$
\|x+y\|^{2} \leq\|x\|^{2}+2\langle y, x+y\rangle, \quad \forall x, y \in H
$$

The lemma is a basic result of a Hilbert space with the inner product.

Lemma 2 (demiclosedness principle). Assume that $T$ is a nonexpansive self-mapping on a nonempty closed convex subset $K$ of a Hilbert space $H$. If $T$ has a fixed point, then $(I-T)$ is demiclosed. That is, whenever $x_{n}$ is a sequence in $K$ weakly converging to some $x \in K$ and the sequence $(I-T) x_{n}$ strongly converges to some $y \in H$, it follows that $(I-T) x=y$. Here $I$ is the identity operator of $H$.

The following lemma is an immediate result of a projection mapping onto a closed convex subset of a Hilbert space.

Lemma 3. Let $K$ be a nonempty closed convex subset of $H$. For all $x, y \in H$ and $z \in K$, then
(1) $\left\langle P_{K}(x)-x, z-P_{K}(y)\right\rangle \geq 0$,
(2) $\left\|P_{K}(x)-P_{K}(y)\right\|^{2} \leq 1\|x-y\|^{2}-\left\|P_{K}(x)-x+y-P_{K}(y)\right\|^{2}$.

Lemma 4 (see [13]). Let $\left\{x_{n}\right\}$ and $\left\{y_{n}\right\}$ be bounded sequence in a Banach space $X$ and let $\left\{\zeta_{n}\right\}$ be a sequence in $[0,1]$ with $0<\liminf _{n \rightarrow \infty} \zeta_{n} \leq \lim \sup _{n \rightarrow \infty} \zeta_{n}<1$. Suppose $x_{n+1}=$ $\left(1-\zeta_{n}\right) y_{n}+\zeta_{n} x_{n}$ for all integers $n \geq 0$ and $\lim \sup _{n \rightarrow \infty}\left(\| y_{n+1}-\right.$ $\left.y_{n}\|-\| x_{n+1}-x_{n} \|\right) \leq 0$. Then $\lim \sup _{n \rightarrow \infty}\left\|y_{n}-x_{n}\right\|=0$.

Lemma $5([5,7])$. Let $\left\{s_{n}\right\}$ be a sequence of nonnegative real numbers satisfying the inequality

$$
s_{n+1} \leq\left(1-\alpha_{n}\right) s_{n}+\alpha_{n} \tau_{n}+\gamma_{n}, \quad \forall n \geq 0,
$$

where $\alpha_{n}, \tau_{n}$, and $\gamma_{n}$ satisfy the following conditions:

(1) $\alpha_{n} \subset[0,1], \sum_{n=0}^{\infty} \alpha_{n}=\infty$, or $\prod_{n=0}^{\infty}\left(1-\alpha_{n}\right)=0$,

(2) $\lim _{n \rightarrow \infty} \sup \tau_{n} \leq 0$,

(3) $\gamma_{n} \subset[0, \infty), \sum_{n=0}^{\infty} \gamma_{n}<\infty$.

Then $\lim _{n \rightarrow \infty} s_{n}=0$.

Since $F$ is $\eta$-strongly monotone, $\operatorname{VI}(F, K)$ has a unique solution $x^{*} \in K$ [5]. Assume that $T: H \rightarrow H$ is a nonexpansive mapping with the fixed point set $\operatorname{Fix}(T)=K$. Obviously $\operatorname{Fix}\left(P_{K}\right)=K$.

For any given numbers $\lambda \in(0,1)$ and $\mu \in\left(0,2 \eta / \kappa^{2}\right)$, we define the mapping $T_{\mu}^{\lambda}: H \rightarrow H$ by

$$
T_{\mu}^{\lambda} x: T x-\lambda \mu F(T x), \quad \forall x \in H .
$$

Lemma 6 (see [5]). If $0<\mu<2 \eta / \kappa^{2}$ and $0<\lambda<1$, then $T_{\mu}^{\lambda}$ is a contraction. In fact,

$$
\left\|T_{\mu}^{\lambda} x-T_{\mu}^{\lambda} y\right\| \leq(1-\lambda \delta)\|x-y\|,
$$

where $\delta=1-\sqrt{1-\mu\left(2 \eta-\mu \kappa^{2}\right)}$, for all $x, y \in H$.

Lemma 7 (see [7]). Let $\left\{\alpha_{n}\right\}$ be a sequence of nonnegative numbers with $\limsup _{n \rightarrow \infty} \alpha_{n}<\infty$ and let $\left\{\beta_{n}\right\}$ be sequence of real numbers with $\lim \sup _{n \rightarrow \infty} \beta_{n} \leq 0$. Then

$$
\lim _{n \rightarrow \infty} \sup _{n} \beta_{n} \leq 0 \text {. }
$$

\section{Convergence Theorem}

Before analyzing the convergence theorem, we first review the $\mathrm{PRH}$ method and related results [15].

Algorithm 8 (see [15]). Take three fixed numbers $t, \rho, \gamma \in$ $\left(0,2 \eta / \kappa^{2}\right)$, starting with arbitrarily chosen initial points $x_{0} \in$ $H$, compute the sequences $\left\{x_{n}\right\},\left\{\bar{x}_{n}\right\},\left\{\tilde{x}_{n}\right\},\left\{\widehat{x}_{n}\right\}$ such that;

Prediction

Step 1: $\bar{x}_{n}=\gamma_{n} x_{n}+\left(1-\gamma_{n}\right)\left[T x_{n}-\lambda_{n+1}^{\prime \prime} \gamma F\left(T x_{n}\right)\right]$, Step 2: $\tilde{x}_{n}=\beta_{n} x_{n}+\left(1-\beta_{n}\right)\left[T \bar{x}_{n}-\lambda_{n+1}^{\prime} \rho F\left(T \bar{x}_{n}\right)\right]$, Step 3: $\widehat{x}_{n}=\theta_{n} \bar{x}_{n}+\left(1-\theta_{n}\right) \tilde{x}_{n}, 0 \leq \theta_{n} \leq 1$, 


\section{Correction}

$$
\begin{aligned}
& \text { Step 4: } x_{n+1}=\alpha_{n} \bar{x}_{n}+\left(1-\alpha_{n}\right)\left[T \widehat{x}_{n}\right. \\
& \left.-\lambda_{n+1} t F\left(T \widehat{x}_{n}\right)\right]
\end{aligned}
$$

where $T: H \rightarrow H$ is a nonexpansive mapping.

Let $\left\{\alpha_{n}\right\} \subset[0,1),\left\{\beta_{n}\right\} \subset[0,1]$ and $\left\{\gamma_{n}\right\} \subset[0,1],\left\{\lambda_{n}\right\},\left\{\lambda_{n}^{\prime}\right\}$, $\left\{\lambda_{n}^{\prime \prime}\right\} \subset(0,1)$ satisfy the following conditions.

Remark 9. In fact, the PRH method is the MRHSD method when $\theta_{n} \equiv 0$, for all $n$.

Condition 10. One has

(1) $\quad \sum_{1}^{\infty}\left|\alpha_{n}-\alpha_{n-1}\right|<\infty, \quad \sum_{1}^{\infty}\left|\beta_{n}-\beta_{n-1}\right|<\infty$,

$$
\sum_{1}^{\infty}\left|\gamma_{n}-\gamma_{n-1}\right|<\infty
$$

(2) $\quad \lim _{n \rightarrow \infty} \alpha_{n}=0, \quad \lim _{n \rightarrow \infty} \beta_{n}=1, \quad \lim _{n \rightarrow \infty} \gamma_{n}=1$,

(3) $\quad \lim _{n \rightarrow \infty} \lambda_{n}=0, \quad \lim _{n \rightarrow \infty} \frac{\lambda_{n}}{\lambda_{n+1}}=1, \quad \sum_{1}^{\infty} \lambda_{n}=\infty$,

(4) $\lambda_{n} \geq \max \left\{\lambda_{n}^{\prime}, \lambda_{n}^{\prime \prime}\right\}, \quad \forall n \geq 1$.

Theorem 11 (see [15]). In Condition 10, the sequence $\left\{x_{n}\right\}$ converges strongly to $x^{*} \in K$, and $x^{*}$ is the unique solution of the $\operatorname{VI}(F, K)$.

We obtain the strong convergence theorem of $\mathrm{PRH}$ method for variational inequalities under different assumptions.

Condition 12. One has

$$
\begin{aligned}
& \text { (1) } 0<\liminf _{n \rightarrow \infty} \alpha_{n} \leq \limsup _{n \rightarrow \infty} \alpha_{n}<1, \\
& \lim _{n \rightarrow \infty} \beta_{n}=1, \quad \lim _{n \rightarrow \infty} \gamma_{n}=1, \\
& \text { (2) } \quad \lim _{n \rightarrow \infty} \lambda_{n}=0, \quad \sum_{1}^{\infty} \lambda_{n}=\infty, \\
& \text { (3) } \lambda_{n} \geq \max \left\{\lambda_{n}^{\prime}, \lambda_{n}^{\prime \prime}\right\}, \quad \forall n \geq 1 .
\end{aligned}
$$

Theorem 13. The sequence $\left\{x_{n}\right\}$ converges strongly to $x^{*} \in K$, and $x^{*}$ is the unique solution of the $\operatorname{VI}(F, K)$. Assume that $\left\{\alpha_{n}\right\},\left\{\beta_{n}\right\}$ and $\left\{\gamma_{n}\right\},\left\{\lambda_{n}\right\},\left\{\lambda_{n}^{\prime}\right\},\left\{\lambda_{n}^{\prime \prime}\right\}$ satisfy Condition 12 .

Proof. We divide the proof into several steps.

Step 1. $\left\{x_{n}\right\},\left\{\bar{x}_{n}\right\},\left\{\tilde{x}_{n}\right\}$, and $\left\{\widehat{x}_{n}\right\}$ are bounded. Since $F$ is $\eta$ strongly monotone, $\operatorname{VI}(F, K)(1)$ has a unique solution $x^{*} \in$ $K$, and $T_{t}^{\lambda_{n+1}} x^{*}=x^{*}-\lambda_{n+1} t F\left(x^{*}\right), T_{\rho}^{\lambda_{n+1}^{\prime}} x^{*}=x^{*}-\lambda_{n+1} \rho F\left(x^{*}\right)$, $T_{\gamma}^{\lambda_{n+1}^{\prime \prime}} x^{*}=x^{*}-\lambda_{n+1} \gamma F\left(x^{*}\right)$.
A series of computations yields

$$
\begin{aligned}
\left\|x_{n+1}-x^{*}\right\|= & \left\|\alpha_{n} \bar{x}_{n}+\left(1-\alpha_{n}\right) T_{t}^{\lambda_{n+1}} \widehat{x}-x^{*}\right\| \\
\leq & \alpha_{n}\left\|\bar{x}_{n}-x^{*}\right\|+\left(1-\alpha_{n}\right)\left\|T_{t}^{\lambda_{n+1}} \widehat{x}-x^{*}\right\| \\
\leq & \alpha_{n}\left\|\bar{x}_{n}-x^{*}\right\|+\left(1-\alpha_{n}\right) \\
& \times\left[\left\|T_{t}^{\lambda_{n+1}} \widehat{x}-T_{t}^{\lambda_{n+1}} x^{*}\right\|+\left\|T_{t}^{\lambda_{n+1}} x^{*}-x^{*}\right\|\right] \\
\leq & \alpha_{n}\left\|\bar{x}_{n}-x^{*}\right\|+\left(1-\alpha_{n}\right) \\
& \times\left[\left(1-\lambda_{n+1} \tau\right)\left\|\widehat{x}_{n}-x^{*}\right\|+\lambda_{n+1} t\left\|F\left(x^{*}\right)\right\|\right],
\end{aligned}
$$

where $\tau=1-\sqrt{1-t\left(2 \eta-t \kappa^{2}\right)} \in(0,1)$,

$$
\begin{aligned}
\left\|\tilde{x}_{n}-x^{*}\right\|= & \left\|\beta_{n} x_{n}+\left(1-\beta_{n}\right) T_{\rho}^{\lambda_{n+1}^{\prime}} \bar{x}_{n}-x^{*}\right\| \\
\leq & \beta_{n}\left\|x_{n}-x^{*}\right\|+\left(1-\beta_{n}\right)\left\|T_{\rho}^{\lambda_{n+1}^{\prime}} \bar{x}_{n}-x^{*}\right\| \\
\leq & \beta_{n}\left\|x_{n}-x^{*}\right\|+\left(1-\beta_{n}\right) \\
& \times\left[\left\|T_{\rho}^{\lambda_{n+1}^{\prime}} \bar{x}_{n}-T_{\rho}^{\lambda_{n+1}^{\prime}} x^{*}\right\|+\left\|T_{\rho}^{\lambda_{n+1}^{\prime}} x^{*}-x^{*}\right\|\right] \\
\leq & \beta_{n}\left\|x_{n}-x^{*}\right\|+\left(1-\beta_{n}\right) \\
& \times\left[\left(1-\lambda_{n+1}^{\prime} \tau^{\prime}\right)\left\|\bar{x}_{n}-x^{*}\right\|+\lambda_{n+1}^{\prime} \rho\left\|F\left(x^{*}\right)\right\|\right] \\
\leq & \beta_{n}\left\|x_{n}-x^{*}\right\|+\left(1-\beta_{n}\right)\left\|\bar{x}_{n}-x^{*}\right\| \\
& +\left(1-\beta_{n}\right) \lambda_{n+1}^{\prime} \rho\left\|F\left(x^{*}\right)\right\|,
\end{aligned}
$$

where $\tau^{\prime}=1-\sqrt{1-\rho\left(2 \eta-t \kappa^{2}\right)} \in(0,1)$.

Moreover, we also obtain

$$
\begin{aligned}
\left\|\tilde{x}_{n}-x^{*}\right\|= & \left\|\gamma_{n} x_{n}+\left(1-\gamma_{n}\right) T_{\gamma}^{\lambda_{n+1}^{\prime \prime}} x_{n}-x^{*}\right\| \\
\leq & \gamma_{n}\left\|x_{n}-x^{*}\right\|+\left(1-\gamma_{n}\right)\left\|T_{\gamma}^{\lambda_{n+1}^{\prime \prime}} x_{n}-x^{*}\right\| \\
\leq & \gamma_{n}\left\|x_{n}-x^{*}\right\|+\left(1-\gamma_{n}\right) \\
& \times\left[\left\|T_{\gamma}^{\lambda_{n+1}^{\prime \prime}} x_{n}-T_{\gamma}^{\lambda_{n+1}^{\prime \prime}} x^{*}\right\|+\left\|T_{\gamma}^{\lambda_{n+1}^{\prime \prime}} x^{*}-x^{*}\right\|\right] \\
\leq & \gamma_{n}\left\|x_{n}-x^{*}\right\|+\left(1-\gamma_{n}\right) \\
& \times\left[\left(1-\lambda_{n+1}^{\prime \prime} \tau^{\prime \prime}\right)\left\|x_{n}-x^{*}\right\|+\lambda_{n+1}^{\prime \prime} \gamma\left\|F\left(x^{*}\right)\right\|\right] \\
\leq & \left\|x_{n}-x^{*}\right\|+\left(1-\gamma_{n}\right) \lambda_{n+1}^{\prime \prime} \gamma\left\|F\left(x^{*}\right)\right\|
\end{aligned}
$$


where $\tau^{\prime \prime}=1-\sqrt{1-\gamma\left(2 \eta-t \kappa^{2}\right)} \in(0,1)$, subtituting; (14) into (13) and (14) into (12), we immediately obtain

$$
\begin{aligned}
\left\|\tilde{x}_{n}-x^{*}\right\|= & \beta_{n}\left\|x_{n}-x^{*}\right\|+\left(1-\beta_{n}\right) \\
& \times\left[\left(1-\lambda_{n+1}^{\prime} \tau^{\prime}\right)\left\|\bar{x}_{n}-x^{*}\right\|+\lambda_{n+1}^{\prime} \rho\left\|F\left(x^{*}\right)\right\|\right] \\
\leq & \beta_{n}\left\|x_{n}-x^{*}\right\|+\left(1-\beta_{n}\right) \\
& \times\left[\left(1-\lambda_{n+1}^{\prime} \tau^{\prime}\right)\left\|x_{n}-x^{*}\right\|\right. \\
& \left.\quad+\left(1-\gamma_{n}\right) \lambda_{n+1}^{\prime \prime} \gamma\left\|F\left(x^{*}\right)\right\|+\lambda_{n+1}^{\prime} \rho\left\|F\left(x^{*}\right)\right\|\right] \\
\leq & \left\|x_{n}-x^{*}\right\|+\left(1-\beta_{n}\right) \lambda_{n+1}(\gamma+\rho)\left\|F\left(x^{*}\right)\right\| .
\end{aligned}
$$

Furthermore,

$$
\begin{aligned}
& \left\|\widehat{x}_{n}-x^{*}\right\|=\left\|\theta_{n} \bar{x}_{n}+\left(1-\theta_{n}\right) \tilde{x}_{n}-x^{*}\right\| \\
& \leq \theta_{n}\left\|\bar{x}_{n}-x^{*}\right\|+\left(1-\theta_{n}\right)\left\|\widetilde{x}_{n}-x^{*}\right\| \\
& \leq \theta_{n}\left[\left\|x_{n}-x^{*}\right\|+\left(1-\gamma_{n}\right) \lambda_{n+1}^{\prime \prime} \gamma\left\|F\left(x^{*}\right)\right\|\right] \\
& +\left(1-\theta_{n}\right)\left[\left\|x_{n}-x^{*}\right\|+\left(1-\beta_{n}\right) \lambda_{n+1}\right. \\
& \left.\times(\gamma+\rho)\left\|F\left(x^{*}\right)\right\|\right] \\
& \leq\left\|x_{n}-x^{*}\right\|+\left(1-\gamma_{n}\right) \lambda_{n+1}^{\prime \prime} \gamma\left\|F\left(x^{*}\right)\right\| \\
& +\left(1-\beta_{n}\right) \lambda_{n+1}(\gamma+\rho)\left\|F\left(x^{*}\right)\right\|, \\
& \left\|x_{n+1}-x^{*}\right\| \\
& \leq \alpha_{n}\left\|\bar{x}_{n}-x^{*}\right\|+\left(1-\alpha_{n}\right) \\
& \times\left[\left(1-\lambda_{n+1} \tau\right)\left\|\widehat{x}_{n}-x^{*}\right\|+\lambda_{n+1} t\left\|F\left(x^{*}\right)\right\|\right] \\
& \leq \alpha_{n}\left[\left\|x_{n}-x^{*}\right\|+\left(1-\gamma_{n}\right) \lambda_{n+1}^{\prime \prime} \gamma\left\|F\left(x^{*}\right)\right\|\right] \\
& +\left(1-\alpha_{n}\right)\left\{\left(1-\lambda_{n+1} \tau\right)\right. \\
& \times\left[\left\|x_{n}-x^{*}\right\|+\left(1-\gamma_{n}\right) \lambda_{n+1}^{\prime \prime} \gamma\left\|F\left(x^{*}\right)\right\|\right. \\
& \left.+\left(1-\beta_{n}\right) \lambda_{n+1}(\gamma+\rho)\left\|F\left(x^{*}\right)\right\|\right] \\
& \left.+\lambda_{n+1} t\left\|F\left(x^{*}\right)\right\|\right\} \\
& \leq \alpha_{n}\left\|x_{n}-x^{*}\right\|+\alpha_{n}\left(1-\gamma_{n}\right) \lambda_{n+1} \gamma\left\|F\left(x^{*}\right)\right\|+\left(1-\alpha_{n}\right) \\
& \times\left[\left(1-\lambda_{n+1} \tau\right)\left\|x_{n}-x^{*}\right\|+\lambda_{n+1}(2 \gamma+\rho+t)\left\|F\left(x^{*}\right)\right\|\right] \text {. }
\end{aligned}
$$

It is easy to obtain the following by induction:

$$
\left\|x_{n}-x^{*}\right\| \leq M_{0}, \quad \forall n \geq 0,
$$

where $M_{0}=\max \left\{3\left\|x_{0}-x^{*}\right\|, 3(\rho+\gamma+t)\left\|F\left(x^{*}\right)\right\| / \tau\right\}$,

$$
\begin{aligned}
\left\|\widetilde{x}_{n}-x^{*}\right\| & \leq\left\|x_{n}-x^{*}\right\|+\left(1-\beta_{n}\right) \lambda_{n+1}(\gamma+\rho)\left\|F\left(x^{*}\right)\right\| \\
& \leq\left(1+\frac{\tau}{3}\right) M_{0}, \\
\left\|\bar{x}_{n}-x^{*}\right\| & \leq\left\|x_{n}-x^{*}\right\|+\left(1-\gamma_{n}\right) \lambda_{n+1}^{\prime \prime} \gamma\left\|F\left(x^{*}\right)\right\| \\
& \leq\left(1+\frac{\tau}{3}\right) M_{0}, \\
\left\|\widehat{x}_{n}-x^{*}\right\| & \leq \theta_{n}\left\|\bar{x}_{n}-x^{*}\right\|+\left(1-\theta_{n}\right)\left\|\widetilde{x}_{n}-x^{*}\right\| \\
& \leq 2\left(1+\frac{\tau}{3}\right) M_{0} .
\end{aligned}
$$

Hence

$$
\begin{aligned}
& \left\{T x_{n}\right\},\left\{T \bar{x}_{n}\right\},\left\{T \widetilde{x}_{n}\right\},\left\{T \widehat{x}_{n}\right\}, \\
& \left\{F\left(T x_{n}\right)\right\},\left\{F\left(T \bar{x}_{n}\right)\right\},\left\{F\left(T \widetilde{x}_{n}\right)\right\},\left\{F\left(T \widehat{x}_{n}\right)\right\}
\end{aligned}
$$

are also bounded.

Step 2. Consider $\left\|x_{n+1}-x_{n}\right\| \rightarrow 0$.

Indeed, by a series of computations, we have

$$
\begin{aligned}
\| \bar{x}_{n}- & \bar{x}_{n-1} \| \\
= & \| \gamma_{n} x_{n}-\gamma_{n-1} x_{n-1}+\left(1-\gamma_{n}\right) T_{\gamma}^{\lambda_{n+1}^{\prime \prime}} x_{n} \\
& \quad-\left(1-\gamma_{n-1}\right) T_{\gamma}^{\lambda_{n}^{\prime \prime}} x_{n-1} \| \\
\leq & \left\|\gamma_{n} x_{n}-\gamma_{n-1} x_{n-1}\right\| \\
& +\left\|\left(1-\gamma_{n}\right) T_{\gamma}^{\lambda_{n+1}^{\prime \prime}} x_{n}-\left(1-\gamma_{n-1}\right) T_{\gamma}^{\lambda_{n}^{\prime \prime}} x_{n-1}\right\| \\
\leq & \left\|x_{n}-x_{n-1}\right\|+\left|\left(1-\gamma_{n}\right) \lambda_{n+1}^{\prime \prime}-\left(1-\gamma_{n-1}\right) \lambda_{n}^{\prime \prime}\right| \\
& \times \gamma\left\|F\left(T x_{n-1}\right)\right\| \\
& +\left|\gamma_{n}-\gamma_{n-1}\right|\left(\left\|x_{n-1}\right\|+\left\|T x_{n-1}\right\|\right) .
\end{aligned}
$$

According to (20) and the prediction step of Algorithm 8, we also obtain

$$
\begin{aligned}
\| \tilde{x}_{n}- & \tilde{x}_{n-1} \| \\
= & \| \beta_{n} x_{n}-\beta_{n-1} x_{n-1}+\left(1-\beta_{n}\right) T_{\rho}^{\lambda_{n+1}^{\prime}} \bar{x}_{n} \\
& \quad-\left(1-\beta_{n-1}\right) T_{\rho}^{\lambda_{n}^{\prime} \bar{x}_{n-1} \|} \\
\leq & \left\|\beta_{n} x_{n}-\beta_{n-1} x_{n-1}\right\| \\
& +\left\|\left(1-\beta_{n}\right) T_{\rho}^{\lambda_{n+1}^{\prime}} \bar{x}_{n}-\left(1-\beta_{n-1}\right) T_{\rho}^{\lambda_{n}^{\prime}} \bar{x}_{n-1}\right\|
\end{aligned}
$$




$$
\begin{aligned}
\leq & \left\|x_{n}-x_{n-1}\right\|+\left|\left(1-\beta_{n}\right) \lambda_{n+1}^{\prime}-\left(1-\beta_{n-1}\right) \lambda_{n}^{\prime}\right| \\
& \times \rho\left\|F\left(T \bar{x}_{n-1}\right)\right\|+\left(1-\beta_{n}\right)\left(1-\lambda_{n+1}^{\prime} \tau^{\prime}\right) \\
& \times\left|\gamma_{n}-\gamma_{n-1}\right|\left(\left\|x_{n-1}\right\|+\left\|T x_{n-1}\right\|\right) \\
& +\left(1-\beta_{n}\right)\left(1-\lambda_{n+1}^{\prime} \tau^{\prime}\right)\left|\left(1-\gamma_{n}\right) \lambda_{n+1}^{\prime \prime}-\gamma_{n-1} \lambda_{n}^{\prime \prime}\right| \\
& \times \gamma\left\|F\left(T x_{n-1}\right)\right\|+\left|\beta_{n}-\beta_{n-1}\right| \\
& \times\left(\left\|x_{n-1}\right\|+\left\|T \bar{x}_{n-1}\right\|+\left\|T \bar{x}_{n-1}\right\|\right) .
\end{aligned}
$$

Also by the prediction step of Algorithm 8 and (20), (21), we have

$$
\begin{aligned}
\| \widehat{x}_{n} & -\widehat{x}_{n-1}\left\|\leq \theta_{n}\right\| \bar{x}_{n}-\bar{x}_{n-1}\left\|+\left(1-\theta_{n}\right)\right\| \tilde{x}_{n}-\tilde{x}_{n-1} \| \\
\leq & \left\|x_{n}-x_{n-1}\right\| \\
& +\left|\left(1-\gamma_{n}\right) \lambda_{n+1}^{\prime \prime}-\left(1-\gamma_{n-1}\right) \lambda_{n}^{\prime \prime}\right| \gamma\left\|F\left(T x_{n-1}\right)\right\| \\
& +\left|\gamma_{n}-\gamma_{n-1}\right|\left(\left\|x_{n-1}\right\|+\left\|T x_{n-1}\right\|\right) \\
& +\left|\left(1-\beta_{n}\right) \lambda_{n+1}^{\prime}-\left(1-\beta_{n-1}\right) \lambda_{n}^{\prime}\right| \rho\left\|F\left(T \bar{x}_{n-1}\right)\right\| \\
& +\left(1-\beta_{n}\right)\left(1-\lambda_{n+1}^{\prime} \tau^{\prime}\right)\left|\gamma_{n}-\gamma_{n-1}\right| \\
& \times\left(\left\|x_{n-1}\right\|+\left\|T x_{n-1}\right\|\right)+\left(1-\beta_{n}\right)\left(1-\lambda_{n+1}^{\prime} \tau^{\prime}\right) \\
& \times\left|\left(1-\gamma_{n}\right) \lambda_{n+1}^{\prime \prime}-\gamma_{n-1} \lambda_{n}^{\prime \prime}\right| \gamma\left\|F\left(T x_{n-1}\right)\right\| \\
& +\left|\beta_{n}-\beta_{n-1}\right|\left(\left\|x_{n-1}\right\|+\left\|T \bar{x}_{n-1}\right\|+\left\|T \bar{x}_{n-1}\right\|\right) .
\end{aligned}
$$

Let

$$
\widehat{y}_{n}=T_{t}^{\lambda_{n+1}} \widehat{x}_{n}=T \widehat{x}_{n}-\lambda_{n+1} t F\left(T \widehat{x}_{n}\right),
$$

so we get

$$
x_{n+1}=\alpha_{n} \bar{x}_{n}+\left(1-\alpha_{n}\right) \hat{y}_{n} .
$$

Furthermore,

$$
\begin{aligned}
\left\|\widehat{y}_{n}-\widehat{y}_{n-1}\right\| \\
=\left\|T \widehat{x}_{n}-T \widehat{x}_{n-1}+\lambda_{n} t F\left(T \widehat{x}_{n-1}\right)-\lambda_{n+1} t F\left(T \widehat{x}_{n}\right)\right\| \\
\leq\left\|T \widehat{x}_{n}-T \widehat{x}_{n-1}\right\|+\lambda_{n} t\left\|F\left(T \widehat{x}_{n-1}\right)\right\| \\
\quad+\lambda_{n+1} t\left\|F\left(T \widehat{x}_{n}\right)\right\| \\
\leq\left\|\widehat{x}_{n}-\widehat{x}_{n-1}\right\|+\lambda_{n} t\left\|F\left(T \widehat{x}_{n-1}\right)\right\| \\
\quad+\lambda_{n+1} t\left\|F\left(T \widehat{x}_{n}\right)\right\| .
\end{aligned}
$$

Apply $\lim _{n \rightarrow \infty} \beta_{n}=1, \lim _{n \rightarrow \infty} \lambda_{n}=0$, and $\lim _{n \rightarrow \infty} \gamma_{n}=1$ and (22), (25) to get

$$
\begin{aligned}
\| \widehat{y}_{n}- & \hat{y}_{n-1}\|-\| x_{n}-x_{n-1} \| \\
\leq & \left|\left(1-\gamma_{n}\right) \lambda_{n+1}^{\prime \prime}-\left(1-\gamma_{n-1}\right) \lambda_{n}^{\prime \prime}\right| \gamma\left\|F\left(T x_{n-1}\right)\right\| \\
& +\left|\gamma_{n}-\gamma_{n-1}\right|\left(\left\|x_{n-1}\right\|+\left\|T x_{n-1}\right\|\right) \\
& +\left|\left(1-\beta_{n}\right) \lambda_{n+1}^{\prime}-\left(1-\beta_{n-1}\right) \lambda_{n}^{\prime}\right| \rho\left\|F\left(T \bar{x}_{n-1}\right)\right\| \\
& +\left(1-\beta_{n}\right)\left(1-\lambda_{n+1}^{\prime} \tau^{\prime}\right)\left|\gamma_{n}-\gamma_{n-1}\right|\left(\left\|x_{n-1}\right\|+\left\|T x_{n-1}\right\|\right) \\
& +\left(1-\beta_{n}\right)\left(1-\lambda_{n+1}^{\prime} \tau^{\prime}\right) \\
& \times\left|\left(1-\gamma_{n}\right) \lambda_{n+1}^{\prime \prime}-\gamma_{n-1} \lambda_{n}^{\prime \prime}\right| \gamma\left\|F\left(T\left(x_{n-1}\right)\right)\right\| \\
& +\left|\beta_{n}-\beta_{n-1}\right|\left(\left\|x_{n-1}\right\|+\left\|T \bar{x}_{n-1}\right\|+\left\|T \bar{x}_{n-1}\right\|\right) \\
& +\lambda_{n} t\left\|F\left(T \widehat{x}_{n-1}\right)\right\|+\lambda_{n+1} t\left\|F\left(T \widehat{x}_{n}\right)\right\| \longrightarrow 0 .
\end{aligned}
$$

According to Lemma 4, we obtain

$$
\lim _{n \rightarrow \infty}\left\|\widehat{y}_{n-1}-x_{n-1}\right\|=0 .
$$

Furthermore, by $\lim _{n \rightarrow \infty} \gamma_{n}=1$, we also get

$$
\begin{aligned}
& \left\|\bar{x}_{n}-x_{n}\right\| \\
& \quad=\left\|-\left(1-\gamma_{n}\right) x_{n}+\left(1-\gamma_{n}\right)\left[T x_{n}-\lambda_{n+1}^{\prime} \gamma F\left(T x_{n}\right)\right]\right\| \\
& \quad \leq\left(1-\gamma_{n}\right)\left\|x_{n}\right\|+\left(1-\gamma_{n}\right)\left\|T x_{n}\right\|+\lambda_{n+1}^{\prime} \gamma\left\|F\left(T x_{n}\right)\right\| \longrightarrow 0 .
\end{aligned}
$$

By (27), (28) and the correction step of Algorithm 8, we immediately conclude that

$$
\begin{aligned}
& \left\|x_{n}-x_{n-1}\right\| \\
& \quad=\left\|\alpha_{n-1} \bar{x}_{n-1}+\left(1-\alpha_{n-1}\right) \hat{y}_{n-1}-x_{n-1}\right\| \\
& \quad \leq \alpha_{n-1}\left\|\bar{x}_{n-1}-x_{n-1}\right\|+\left(1-\alpha_{n-1}\right)\left\|\hat{y}_{n-1}-x_{n-1}\right\| \longrightarrow 0,
\end{aligned}
$$

so we get

$$
\left\|x_{n+1}-x_{n}\right\| \longrightarrow 0
$$

Step 3. Consider $\left\|x_{n+1}-T x_{n}\right\| \rightarrow 0$.

Indeed, by the prediction step of Algorithm 8, we have

$$
\begin{aligned}
& \left\|\tilde{x}_{n}-x_{n}\right\| \\
& \quad=\left\|-\left(1-\beta_{n}\right) x_{n}+\left(1-\beta_{n}\right)\left[T \bar{x}_{n}-\lambda_{n+1}^{\prime} \rho F\left(T \bar{x}_{n}\right)\right]\right\| \\
& \quad \leq\left(1-\beta_{n}\right)\left\|x_{n}\right\|+\left(1-\beta_{n}\right)\left[\left\|T \bar{x}_{n}\right\|+\left\|\lambda_{n+1}^{\prime} \rho F\left(T \bar{x}_{n}\right)\right\|\right] .
\end{aligned}
$$

According to the assumption $\lim _{n \rightarrow \infty} \beta_{n}=1$ and $\lim _{n \rightarrow \infty} \lambda_{n}=0$, then

$$
\left\|\widetilde{x}_{n}-x_{n}\right\| \longrightarrow 0 .
$$


By (32), we immediately obtain

$$
\left\|\widehat{x}_{n}-x_{n}\right\| \leq \theta_{n}\left\|\bar{x}_{n}-x_{n}\right\|+\left(1-\theta_{n}\right)\left\|\tilde{x}_{n}-x_{n}\right\| \longrightarrow 0 .
$$

By a series of computations, we can get

$$
\begin{aligned}
&\left\|x_{n+1}-T x_{n}\right\| \\
&=\left\|\alpha_{n}\left(\bar{x}_{n}-T x_{n}\right)+\left(1-\alpha_{n}\right)\left(T_{t}^{\lambda_{n+1}} \hat{x}-T x_{n}\right)\right\| \\
& \leq \alpha_{n}\left\|\bar{x}_{n}-T x_{n}\right\|+\left(1-\alpha_{n}\right)\left\|T \widehat{x}_{n}-T x_{n}\right\| \\
&+\left(1-\alpha_{n}\right) \lambda_{n+1} t\left\|F\left(T \widehat{x}_{n}\right)\right\| \\
& \leq \alpha_{n}\left\|\bar{x}_{n}-T x_{n}\right\|+\left\|\widehat{x}_{n}-x_{n}\right\|+\lambda_{n+1} t\|F(T \widehat{x})\| \\
& \leq \alpha_{n}\left\|x_{n+1}-T x_{n}\right\|+\alpha_{n}\left\|\bar{x}_{n}-x_{n+1}\right\| \\
&+\left\|\widehat{x}_{n}-x_{n}\right\|+\lambda_{n+1} t\|F(T \widehat{x})\| .
\end{aligned}
$$

Hence, by (28), (33), and (34), we also obtain

$$
\begin{aligned}
\left\|x_{n+1}-T x_{n}\right\| \leq & \frac{\alpha_{n}}{1-\alpha_{n}}\left\|\bar{x}_{n}-x_{n+1}\right\| \\
& +\frac{\left\|\widehat{x}_{n}-x_{n}\right\|}{1-\alpha_{n}}+\frac{\lambda_{n+1} t\|F(T \widehat{x})\|}{1-\alpha_{n}} \longrightarrow 0 .
\end{aligned}
$$

Using Steps 2 and 3, it is easy to obtain the following corollary.

Corollary 14. Consider $\left\|x_{n}-T x_{n}\right\| \rightarrow 0$.

Applying Steps 2 and 3, one gets

$$
\left\|x_{n+1}-T x_{n}\right\| \longrightarrow 0, \quad\left\|x_{n+1}-x_{n}\right\| \longrightarrow 0,
$$

so it is easy to see that

$$
\left\|x_{n}-T x_{n}\right\| \leq\left\|x_{n+1}-T x_{n}\right\|+\left\|x_{n+1}-x_{n}\right\| \longrightarrow 0 .
$$

Step 4. Consider $\lim _{n \rightarrow \infty} \sup \left\langle-F\left(x^{*}\right), T \widehat{x}_{n}-x^{*}\right\rangle \leq 0$. that

For some $\widehat{x} \in H$, here exits $\left\{T x_{n_{i}}\right\} \rightarrow \widehat{x}$ weakly and such

$$
\begin{aligned}
\lim _{n \rightarrow \infty} & \sup \left\langle-F\left(x^{*}\right), T x_{n}-x^{*}\right\rangle \\
& =\lim _{n \rightarrow \infty} \sup \left\langle-F\left(x^{*}\right), T x_{n_{i}}-x^{*}\right\rangle .
\end{aligned}
$$

According to $\left\{T x_{n_{i}}\right\} \rightarrow \widehat{x}$, we have

$$
\widehat{x} \in \operatorname{Fix}(T)=K \text {. }
$$

By $x^{*}$ being the unique solution of $\operatorname{VI}(F, K)$, we can obtain

$$
\begin{aligned}
\lim _{n \rightarrow \infty} & \sup \left\langle-F\left(x^{*}\right), T x_{n}-x^{*}\right\rangle \\
& =\lim _{n \rightarrow \infty} \sup \left\langle-F\left(x^{*}\right), \widehat{x}-x^{*}\right\rangle \\
& \leq 0 .
\end{aligned}
$$

Since $\left\|T \widehat{x}_{n}-T x_{n}\right\| \leq\left\|\widehat{x}_{n}-x_{n}\right\| \rightarrow 0$, we immediately conclude that

$$
\begin{aligned}
& \lim _{n \rightarrow \infty} \sup \left\langle-F\left(x^{*}\right), T \widehat{x}_{n}-x^{*}\right\rangle \\
& \leq \lim _{n \rightarrow \infty} \sup \left\langle-F\left(x^{*}\right), T \widehat{x}_{n}-T x_{n}\right\rangle \\
& \quad+\lim _{n \rightarrow \infty} \sup \left\langle-F\left(x^{*}\right), T x_{n}-x^{*}\right\rangle \\
& \leq \lim _{n \rightarrow \infty} \sup \left\langle-F\left(x^{*}\right), T x_{n}-x^{*}\right\rangle \\
& \leq 0 .
\end{aligned}
$$

Step 5. By Step 1 and Lemma 1, we have

$$
\begin{aligned}
& \left\|x_{n+1}-x^{*}\right\|^{2} \\
& =\left\|\alpha_{n}\left(\bar{x}_{n}-x^{*}\right)+\left(1-\alpha_{n}\right)\left(T_{t}^{\lambda_{n+1}} \widehat{x}_{n}-x^{*}\right)\right\|^{2} \\
& \leq\left\|\alpha_{n}\left(\bar{x}_{n}-x^{*}\right)\right\|^{2}+\left(1-\alpha_{n}\right) \\
& \times\left\|\left(T_{t}^{\lambda_{n+1}} \widehat{x}_{n}-T_{t}^{\lambda_{n+1}} x^{*}+T_{t}^{\lambda_{n+1}} x^{*}-x^{*}\right)\right\|^{2} \\
& \leq\left\|\alpha_{n}\left(\bar{x}_{n}-x^{*}\right)\right\|^{2}+\left(1-\alpha_{n}\right) \\
& \times\left[\left\|T_{t}^{\lambda_{n+1}} \widehat{x}_{n}-T_{t}^{\lambda_{n+1}} x^{*}\right\|^{2}\right. \\
& \left.+2\left\langle T_{t}^{\lambda_{n+1}} x^{*}-x^{*}, T_{t}^{\lambda_{n+1}} \widehat{x}_{n}-x^{*}\right\rangle\right] \\
& \leq \alpha_{n}\left[\left\|x_{n}-x^{*}\right\|+\left(1-\gamma_{n}\right) \lambda_{n+1}^{\prime \prime} \gamma\left\|F\left(x^{*}\right)\right\|\right]^{2} \\
& +\left(1-\alpha_{n}\right)\left(1-\lambda_{n+1} \tau\right)^{2} \\
& \times\left[\left\|x_{n}-x^{*}\right\|+\left(1-\gamma_{n}\right) \lambda_{n+1}^{\prime \prime} \gamma\left\|F\left(x^{*}\right)\right\|\right. \\
& \left.+\left(1-\beta_{n}\right) \lambda_{n+1}(\gamma+\rho)\left\|F\left(x^{*}\right)\right\|\right]^{2} \\
& +2 t \lambda_{n+1}\left\langle-F\left(x^{*}\right), T \widehat{x}_{n}-x^{*}-t \lambda_{n+1} F\left(T \widehat{x}_{n}\right)\right\rangle \\
& \leq \alpha_{n}\left\|x_{n}-x^{*}\right\|^{2}+\left(1-\gamma_{n}\right) \lambda_{n+1} \gamma M \\
& +\left(1-\alpha_{n}\right)\left(1-\lambda_{n+1} \tau\right)^{2}\left\|x_{n}-x^{*}\right\|^{2} \\
& +\left(1-\alpha_{n}\right)\left(1-\lambda_{n+1} \tau\right)^{2}\left(1-\beta_{n}\right) \lambda_{n+1} M \\
& +\left(1-\alpha_{n}\right)\left(1-\lambda_{n+1} \tau\right)^{2}\left(1-\gamma_{n}\right) \lambda_{n+1} \gamma M \\
& +2 t \lambda_{n+1}\left\langle-F\left(x^{*}\right), T \hat{x}_{n}-x^{*}-t \lambda_{n+1} F\left(T \hat{x}_{n}\right)\right\rangle \\
& \leq\left[1-\left(1-\alpha_{n}\right) \lambda_{n+1} \tau\right]\left\|x_{n}-x^{*}\right\|^{2} \\
& +\left(1-\alpha_{n}\right) \lambda_{n+1} \tau w_{n+1}^{\prime} \text {, }
\end{aligned}
$$


where

$$
\begin{aligned}
w_{n+1}^{\prime}= & \frac{2 t\left\langle-F\left(x^{*}\right), T \hat{x}_{n}-x^{*}-t \lambda_{n+1} F\left(T \hat{x}_{n}\right)\right\rangle}{\tau\left(1-\alpha_{n}\right)} \\
& +\frac{\varphi_{n}}{\tau\left(1-\alpha_{n}\right)}+\frac{\xi_{n}}{\tau\left(1-\alpha_{n}\right)}, \\
\varphi_{n}= & \left(1-\gamma_{n}\right) \gamma M, \\
\xi_{n}= & \left(1-\alpha_{n}\right)\left(1-\lambda_{n+1} \tau\right)^{2}\left(1-\beta_{n}\right) M \\
& +\left(1-\alpha_{n}\right)\left(1-\lambda_{n+1} \tau\right)^{2}\left(1-\gamma_{n}\right) \lambda_{n+1} \gamma M,
\end{aligned}
$$

and $M_{0} \ll M<\infty$.

Denote

$$
s_{n+1}^{\prime}=\left\|x_{n+1}-x^{*}\right\|, \quad u_{n}=\left(1-\alpha_{n}\right) \lambda_{n+1} \tau .
$$

We can rewrite (42) as

$$
s_{n+1}^{\prime} \leq\left(1-u_{n}\right) s_{n}^{\prime}+u_{n} w_{n}^{\prime}+0 .
$$

In fact, $u_{n}, w_{n}^{\prime}$ satisfies Lemma 5 ; according to

$$
\lim _{n \rightarrow \infty} \beta_{n}=1, \quad \lim _{n \rightarrow \infty} \gamma_{n}=1, \quad \lim _{n \rightarrow \infty} \lambda_{n}=0,
$$

we obtain

$$
\begin{aligned}
& \frac{\varphi_{n}}{\tau\left(1-\alpha_{n}\right)} \longrightarrow 0, \\
& \frac{\xi_{n}}{\tau\left(1-\alpha_{n}\right)} \longrightarrow 0 .
\end{aligned}
$$

Moreover, by Step 4, we also obtain

$$
\begin{aligned}
\lim _{n \rightarrow \infty} & \frac{2 t\left\langle-F\left(x^{*}\right), T \hat{x}_{n}-x^{*}-t \lambda_{n+1} F\left(T \widehat{x}_{n}\right)\right\rangle}{\tau\left(1-\alpha_{n}\right)} \\
\leq & \frac{2 t}{\tau} \lim _{n \rightarrow \infty} \sup \left\{\left\langle-F\left(x^{*}\right), T \widehat{x}_{n}-x^{*}\right\rangle\right. \\
& \left.+\lambda_{n+1}\left\langle-F\left(x^{*}\right),-t F\left(T \hat{x}_{n}\right)\right\rangle\right\} \\
\leq & \frac{2 t}{\tau} \lim _{n \rightarrow \infty} \sup \left\{\left\langle-F\left(x^{*}\right), T \widehat{x}_{n}-x^{*}\right\rangle\right\} \\
& +\lim _{n \rightarrow \infty} \sup \left\{\lambda_{n+1}\left\langle-F\left(x^{*}\right),-t F\left(T \widehat{x}_{n}\right)\right\rangle\right\} \\
\leq & +0=0 .
\end{aligned}
$$

Furthermore, by (43), (47), and (48), it is easy to obtain

$$
\lim _{n \rightarrow \infty} \sup w_{n}^{\prime} \leq 0 .
$$

Consequently apply Lemma 5 to obtain

$$
\left\|x_{n}-x^{*}\right\| \longrightarrow 0
$$

\section{Numerical Experiments}

The problem considered in this section is

$$
\min \left\{\frac{1}{2}\|X-C\|_{F}^{2} \mid X \in K\right\},
$$

where $\|\cdot\|_{F}$ is the matrix Fröbenis norm; that is,

$$
\|C\|_{F}=\left(\sum_{i=1}^{\infty} \sum_{j=1}^{\infty}\left|C_{i j}\right|^{2}\right)^{1 / 2} .
$$

Note that the matrix Fröbenis norm is induced by the inner product

$$
\langle A, B\rangle=\operatorname{Trace}\left(A^{T} B\right) .
$$

The problems arise from finance and statistics, and we form the test problems similarly as in $[9,21]$.

Let $K=S_{+}^{n} \cap \boldsymbol{B}$, where

$$
\begin{gathered}
S_{+}^{n}=\left\{H \in \mathbb{R}^{n \times n} \mid H^{T}=H, H \geq 0\right\}, \\
\boldsymbol{B}=\left\{H \in \mathbb{R}^{n \times n} \mid H^{T}=H, H_{L} \leq H \leq H_{U}\right\} .
\end{gathered}
$$

Let $H_{L}, H_{U}$ be given $n \times n$ symmetric matrices, and $C$ asymmetric which differs from previous approaches $[9,21]$, and it is to be noted that the extended contraction method (EC method) [9] has much difficulty in computing the examples when $C$ is asymmetric, where $H_{L} \leq H_{U}$ in element wise:

$$
H_{L} \leq H_{U}:\left(H_{L}\right)_{i j} \leq\left(H_{U}\right)_{i j}, \quad \forall i, j \in 1, \ldots, n .
$$

Then (51) is equivalent to the following variational inequality:

$$
\left\langle X^{\prime}-X, \nabla\left(\frac{1}{2}\|X-C\|^{2}\right)\right\rangle \geq 0, \quad \forall X^{\prime} \in K .
$$

So we get

$$
\left\langle X^{\prime}-X, X-C\right\rangle \geq 0, \quad \forall X^{\prime} \in K .
$$

According to Condition 10, we take the following parameter sequences, and let Condition 10 denote the parameter sequences:

$$
\begin{gathered}
\alpha_{n}=\frac{1}{\ln n}, \\
\lambda_{n}=\lambda_{n}^{\prime}=\lambda_{n}^{\prime \prime}=\frac{1}{\ln (n+1)}, \\
\beta_{n}=\gamma_{n}=1-\frac{1}{\ln n}, \\
\gamma=\rho=t=c_{0}>0 .
\end{gathered}
$$

According to Condition 12, we take the following parameter sequences, and let Condition 12 denote the parameter sequences:

$$
\begin{aligned}
& \alpha_{n}=0.8-\frac{1}{(10 * \ln n)}, \quad n=2 k, \\
& \alpha_{n}=0.3-\frac{1}{(10 * \ln n)}, \quad n=2 k-1,
\end{aligned}
$$


TABLE 1: Numerical results for the PRH method and the EC method.

\begin{tabular}{|c|c|c|c|c|c|c|c|}
\hline \multirow{3}{*}{$\begin{array}{l}\text { Asymmetric matrix } \\
n\end{array}$} & \multicolumn{4}{|c|}{$c_{0}=0.1, \theta_{n}=0.8$, tolerance $=10^{-4}$} & \multirow[b]{3}{*}{ It } & \multirow{2}{*}{\multicolumn{2}{|c|}{ EC method }} \\
\hline & \multicolumn{2}{|c|}{ Condition 10} & \multicolumn{2}{|c|}{ Condition 12} & & & \\
\hline & It & $\mathrm{cpu}$ & It & $\mathrm{cpu}$ & & $\mathrm{cpu}$ & tolerance \\
\hline 100 & 201 & 8.34 & 130 & 5.35 & 100 & 14.46 & $8.289 e+000$ \\
\hline 200 & 333 & 75.44 & 208 & 47.14 & 100 & 94.30 & $1.010 e+002$ \\
\hline 300 & 443 & 318.02 & 272 & 174.70 & 100 & 302.29 & $4.899 e+002$ \\
\hline 400 & 543 & 789.16 & 330 & 446.00 & 100 & 686.83 & $9.628 e+002$ \\
\hline 500 & 647 & 1747.70 & 388 & 972.18 & 100 & 1287.36 & $1.756 e+003$ \\
\hline 1000 & 1082 & 19884.30 & 634 & 11502.13 & 100 & 9220.50 & $9.826 e+003$ \\
\hline 2000 & $>2000$ & $>150000$ & 1052 & 128504.67 & 100 & $>74640.41$ & $>5.597 e+003$ \\
\hline
\end{tabular}

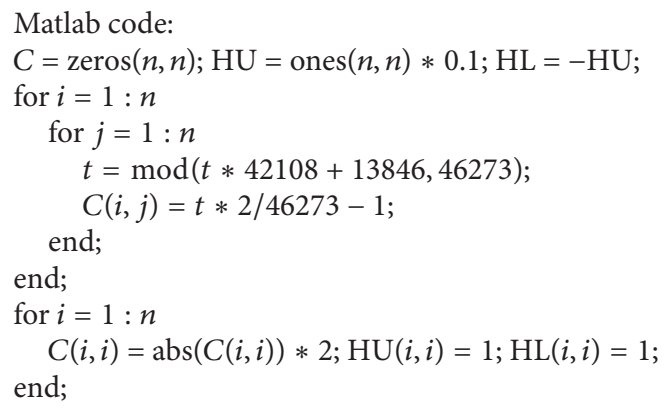

Algorithm 1

TABle 2: Numerical results for tolerance $10^{-4}$.

\begin{tabular}{lcccc}
\hline $\begin{array}{l}\text { Asymmetric } \\
\text { matrix }\end{array}$ & \multicolumn{4}{c}{$c_{0}=0.1, \theta_{n}=0.8$} \\
$n$ & It & condition 10 & It & condition 12 \\
\hline 100 & 204 & 8.78 & 130 & 5.45 \\
200 & 330 & 76.08 & 208 & 47.72 \\
300 & 445 & 323.20 & 272 & 175.89 \\
400 & 548 & 867.56 & 330 & 450.59 \\
500 & 663 & 1916.90 & 388 & 994.18 \\
\hline
\end{tabular}

TABLE 3: Numerical results for tolerance $10^{-3}$.

\begin{tabular}{lcccc}
\hline $\begin{array}{l}\text { Asymmetric } \\
\text { matrix }\end{array}$ & \multicolumn{4}{c}{$\mathcal{c}_{0}=0.1, \theta_{n}=0.8$} \\
$n$ & Condition 10 & \multicolumn{2}{c}{ Condition 12} \\
$n$ & It & cpu & It & cpu \\
\hline 1000 & 193 & 3893.63 & 126 & 2280.74 \\
2000 & 318 & 42981.02 & 200 & 28737.65 \\
\hline
\end{tabular}

$$
\begin{aligned}
& \lambda_{n}=\lambda_{n}^{\prime}=\lambda_{n}^{\prime \prime}=\frac{1}{\ln (n+1)}, \\
& \beta_{n}=1-\frac{1}{\ln n}, \quad n=2 k, \\
& \beta_{n}=1-\frac{1}{\ln n}, \quad n=2 k-1,
\end{aligned}
$$

$$
\begin{gathered}
\gamma_{n}=1-\frac{1}{\ln n}, \quad n=2 k, \\
\gamma_{n}=1-\frac{1}{\ln (2 n)}, \quad n=2 k-1, \\
\gamma=\rho=t=c_{0}>0 .
\end{gathered}
$$

Obviously, we have much difficulty in computing the projection of $P_{K}[X]$, for all $x \in S^{n}$. In order to reduce the difficulty and complexity of computing the projection $P_{K}$, we define $T X$ by

$$
T X=H(G(X)),
$$

where

$$
\begin{gathered}
G(X)=\min \left(H_{U}, \max \left(X, H_{L}\right)\right), \\
H(X)=P_{S_{+}^{n}}(X),
\end{gathered}
$$

which can be computed without difficulty and the fixed point set of $\operatorname{Fix}(T)=K$. According to Theorems 11 and 13, the sequences generated by Algorithm 8 under Conditions 10 and 12 are convergent.

The computation begins with ones $(n, n)$ in MATLAB and stops as soon as $\left\|x_{k+1}-x_{k}\right\| \leq 10^{-3}$ or $10^{-2}$. All codes were implemented in MATLAB 7.1 and ran at a Pentium R 1.70G processor, 2G Acer note computer.

We test the problems with $n=100,200,300,400,500$, 1000 , and 2000. The test results with the PRH method under 
TABLE 4: Numerical results for tolerance $10^{-4}$.

\begin{tabular}{|c|c|c|c|c|c|c|c|c|c|c|}
\hline \multirow{3}{*}{$\begin{array}{l}\text { Asymmetric matrix } \\
n\end{array}$} & \multicolumn{10}{|c|}{$\gamma=0.1, \rho=0.3, t=0.1$} \\
\hline & \multicolumn{2}{|c|}{$\theta_{n}=0$} & \multicolumn{2}{|c|}{$\theta_{n}=0.2$} & \multicolumn{2}{|c|}{$\theta_{n}=0.4$} & \multicolumn{2}{|c|}{$\theta_{n}=0.6$} & \multicolumn{2}{|c|}{$\theta_{n}=0.8$} \\
\hline & It & cpu & It & cpu & It & cpu & It & $\mathrm{cpu}$ & It & $\mathrm{cpu}$ \\
\hline 100 & 132 & 5.52 & 134 & 5.60 & 128 & 5.50 & 134 & 5.67 & 132 & 5.54 \\
\hline 200 & 210 & 48.04 & 206 & 47.22 & 208 & 48.04 & 204 & 47.15 & 214 & 48.58 \\
\hline 300 & 274 & 177.49 & 268 & 176.08 & 276 & 178.80 & 274 & 177.68 & 276 & 178.84 \\
\hline 400 & 336 & 468.28 & 328 & 445.93 & 336 & 468.20 & 334 & 454.24 & 330 & 453.79 \\
\hline 500 & 392 & 977.79 & 394 & 1012.57 & 378 & 948.44 & 386 & 953.91 & 390 & 971.10 \\
\hline
\end{tabular}

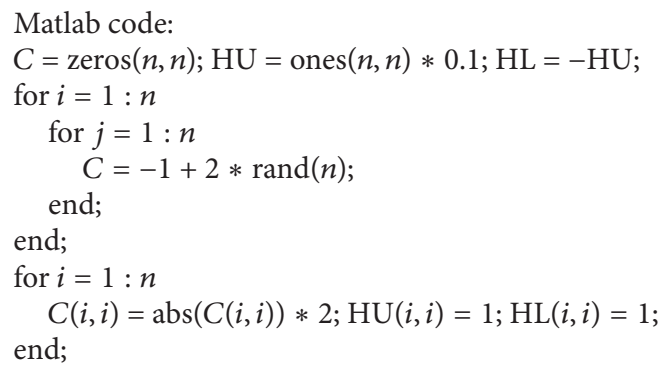

Algorithm 2

different conditions are reported in Tables 1, 2, 3, and 4. And the CPU time is in seconds. It is to be noted that the results of extended contraction method are only given out when the iteration step (It) is less than or equal to 100 .

Test Examples 1. In this example we generate the data in a similar manner as in [9]. The entries of diagonal elements of $C$ are randomly generated in the interval $(0,2)$; the entries of off-diagonal elements of $C$ are randomly generated in the interval $(-1,1)$ (Algorithm 1):

$$
\begin{gathered}
\left(H_{U}\right)_{j j}=\left(H_{L}\right)_{j j}=1, \\
\left(H_{U}\right)_{i j}=-\left(H_{L}\right)_{i j}=0.1, \quad \forall i \neq j, i, j=1,2, \ldots, n .
\end{gathered}
$$

When $n \geq 1000$ and tolerance $10^{-4}$, the computation time of the proposed method is too long, so the results of the PRH method give out approximate solution with $n \geq 1000$ and tolerance $10^{-3}$ in the following. And the extended contraction method (EC method) has much difficulty in computing the examples when $C$ is asymmetric. Furthermore, by introducing auxiliary variable, the certain projection method or relaxed-PPA method [10] can be implemented by these tests.

Test Examples 2. We form the data of the second problems similarly as in the first test examples. The entries of diagonal elements of $C$ are randomly generated in the interval $(0,2)$; the entries of off-diagonal elements of $C$ are generated from a uniform distribution in the same interval (Algorithm 2):

$$
\begin{gathered}
\left(H_{U}\right)_{j j}=\left(H_{L}\right)_{j j}=1, \\
\left(H_{U}\right)_{i j}=-\left(H_{L}\right)_{i j}=0.1, \quad \forall i \neq j, i, j=1,2, \ldots, n .
\end{gathered}
$$

From Tables 1 to 3, we found that the iteration numbers and CPU time of PRH under Condition 12 are more efficient than that under Condition 10. In Table 4 of our method, the tests' results give out that the PRH method under some descent directions is more slightly efficient than those of the MRHSD method $[14,16]$, and it is easy to obtain these descent directions. Furthermore, it is important to find $\gamma, \rho$, and $t$ by Tables 2 and 4 .

\section{Conclusions}

We have proved the strong convergence of PRH method under Condition 12, which differs from Condition 10. The result can be considered as an improvement and refinement of the previous results [14]. And more importantly, numerical experiments demonstrated that the PRH method under Condition 12 is more efficient than that under Condition 10 , and the PRH method under some descent directions is more slightly efficient than that of the MRHSD method. How to select parameters of the PRH method for solving variational inequalities is worthy of further investigations in the future.

\section{Acknowledgments}

This research was supported by National Science and Technology Support Program (Grant no. 2011BAH24B06), Joint Fund of National Natural Science Foundation of China and Civil Aviation Administration of China (Grant no. U1233105), and Science Foundation of the Civil Aviation Flight University of China (Grant no. J2010-45). 


\section{References}

[1] M. S. Gowda and Y. Song, "On semidefinite linear complementarity problems," Mathematical Programming, vol. 88, no. 3, pp. 575-587, 2000.

[2] I. Yamada, "The hybrid steepest descent method for the variational inequality problem over the intersection of fixed point sets of nonexpansive mappings," in Inherently Parallel Algorithms in Feasibility and Optimization and Their Applications, D. Bumariu, Y. Censor, and S. Reich, Eds., vol. 8, pp. 473-504, North-Holland, Amsterdam, The Netherlands, 2001.

[3] F. Deutsch and I. Yamada, "Minimizing certain convex functions over the intersection of the fixed point sets of nonexpansive mappings," Numerical Functional Analysis and Optimization, vol. 19, no. 1-2, pp. 33-56, 1998.

[4] H. K. Xu and T. H. Kim, "Convergence of hybrid steepestdescent methods for variational inequalities," Journal of Optimization Theory and Applications, vol. 119, no. 1, pp. 185-201, 2003.

[5] L. C. Zeng, N. C. Wong, and J. C. Yao, "Convergence analysis of modified hybrid steepest-descent methods with variable parameters for variational inequalities," Journal of Optimization Theory and Applications, vol. 132, no. 1, pp. 51-69, 2007.

[6] L. C. Zeng, "On a general projection algorithm for variational inequalities," Journal of Optimization Theory and Applications, vol. 97, no. 1, pp. 229-235, 1998.

[7] X. P. Ding, Y. C. Lin, and J. C. Yao, "Three-step relaxed hybrid steepest-descent methods for variational inequalities," Applied Mathematics and Mechanics, vol. 28, no. 8, pp. 1029-1036, 2007.

[8] B. S. He, "A new method for a class of linear variational inequalities," Mathematical Programming, vol. 66, no. 2, pp. 137144, 1994.

[9] B. S. He and M. H. Xu, "A general framework of contraction methods for monotone variational inequalities," Pacific Journal of Optimization, vol. 4, no. 2, pp. 195-212, 2008.

[10] B. S. He, "PPA-based contraction methods for general linearly constrained convex optimization," Lectures of Contraction Methods for Convex Optimization and Monotone Variational Inequalities, 06C, 2012, http://math.nju.edu.cn/ hebma/.

[11] N. J. Huang, X. X. Huang, and X. Q. Yang, "Connections among constrained continuous and combinatorial vector optimization," Optimization, vol. 60, no. 1-2, pp. 15-27, 2011.

[12] P. T. Harker and J. S. Pang, "A damped-Newton method for the linear complementarity problem," in Computational Solution of Nonlinear Systems of Equations, vol. 26, pp. 265-284, American Mathematical Society, Providence, RI, USA, 1990.

[13] T. Suzuki, "Strong convergence of Krasnoselskii and Mann's type sequences for one-parameter nonexpansive semigroups without Bochner integrals," Journal of Mathematical Analysis and Applications, vol. 305, no. 1, pp. 227-239, 2005.

[14] H. W. Xu, E. B. Song, H. P. Pan, H. Shao, and L. M. Sun, "The modified and relaxed hybrid steepestdescent methods for variational inequalities," in Proceedings of the 1st International Conference on Modelling and Simulation, vol. 2, pp. 169-174, World Academic Press, 2008.

[15] H. W. Xu, H. Shao, and Q. C. Zhang, "The Prediction-correction and relaxed hybrid steepest-descent method for variational inequalities," in Proceedings of the International Symposium on Education and Computer Science, vol. 1, pp. 252-256, IEEE Computer Society and Academy, 2009.

[16] H. W. Xu, "Efficient implementation of a modified and relaxed hybrid steepest-descent method for a type of variational inequality," Journal of Inequalities and Applications, vol. 2012, article 93, 2012.

[17] J. H. Hammond, Solving asymmetric variational inequality problems and systems of equations with generalized nonlinear programming algorithms [Ph.D. dissertation], Department of Mathematics, MIT, Cambridge, Mass, USA, 1984.

[18] P. Tseng, "Further applications of a splitting algorithm to decomposition in variational inequalities and convex programming," Mathematical Programming, vol. 48, no. 2, pp. 249-263, 1990.

[19] R. A. Horn and C. R. Johnson, Topics in Matrix Analysis, Cambridge University Press, Cambridge, UK, 1991.

[20] D. F. Sun, "A projection and contraction method for generalized nonlinear complementarity problems," Mathematica Numerica Sinica, vol. 16, no. 2, pp. 183-194, 1994.

[21] Y. Gao and D. F. Sun, "Calibrating least squares covariance matrix problems with equality and inequality constraints," Tech. Rep., Department of Mathematics, National University of Singapore, 2008.

[22] M. A. Noor, "Some recent advances in variational inequalities. I. Basic concepts," New Zealand Journal of Mathematics, vol. 26, no. 1, pp. 53-80, 1997.

[23] M. A. Noor, "New approximation schemes for general variational inequalities," Journal of Mathematical Analysis and Applications, vol. 251, no. 1, pp. 217-229, 2000.

[24] Y. Yao, M. A. Noor, R. Chen, and Y.-C. Liou, "Strong convergence of three-step relaxed hybrid steepest-descent methods for variational inequalities," Applied Mathematics and Computation, vol. 201, no. 1-2, pp. 175-183, 2008.

[25] "Advances in Equilibrium Modeling, Analysis, and Computation," in Annals of Operations Research, A. Nagurney, Ed., vol. 44, J. C. Baltzer AG Scientific Publishing, Basel, Switzerland, 1993. 


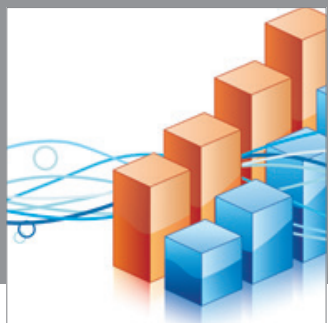

Advances in

Operations Research

mansans

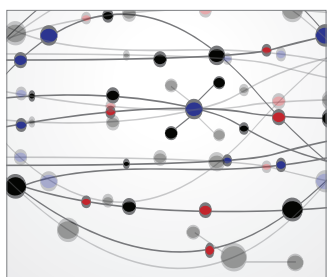

The Scientific World Journal
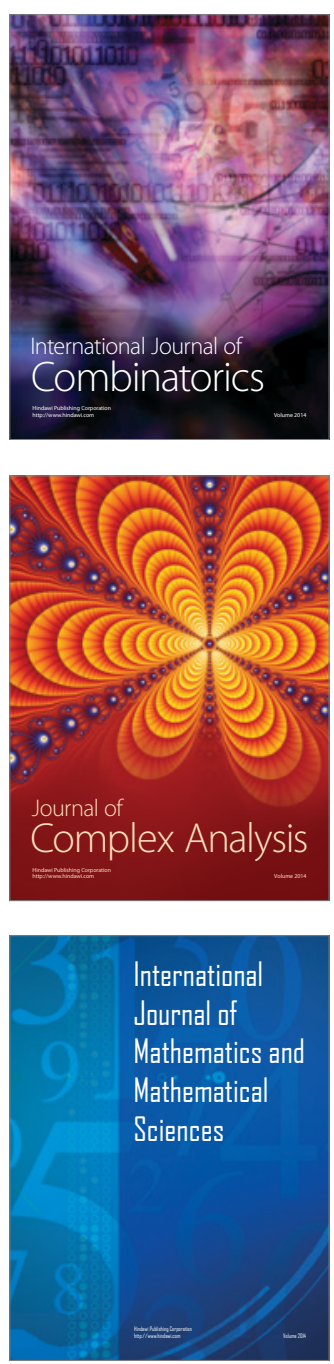
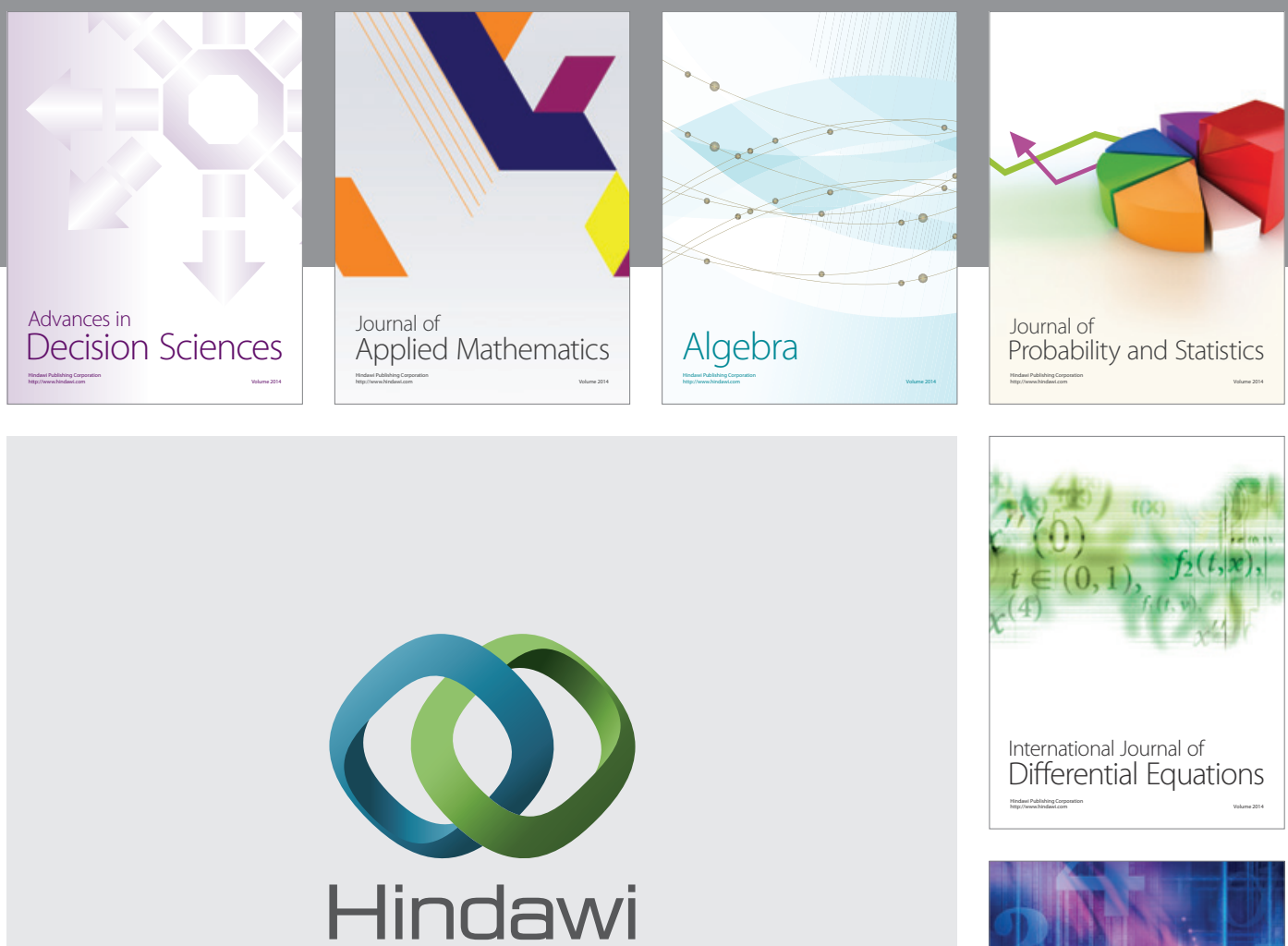

Submit your manuscripts at http://www.hindawi.com
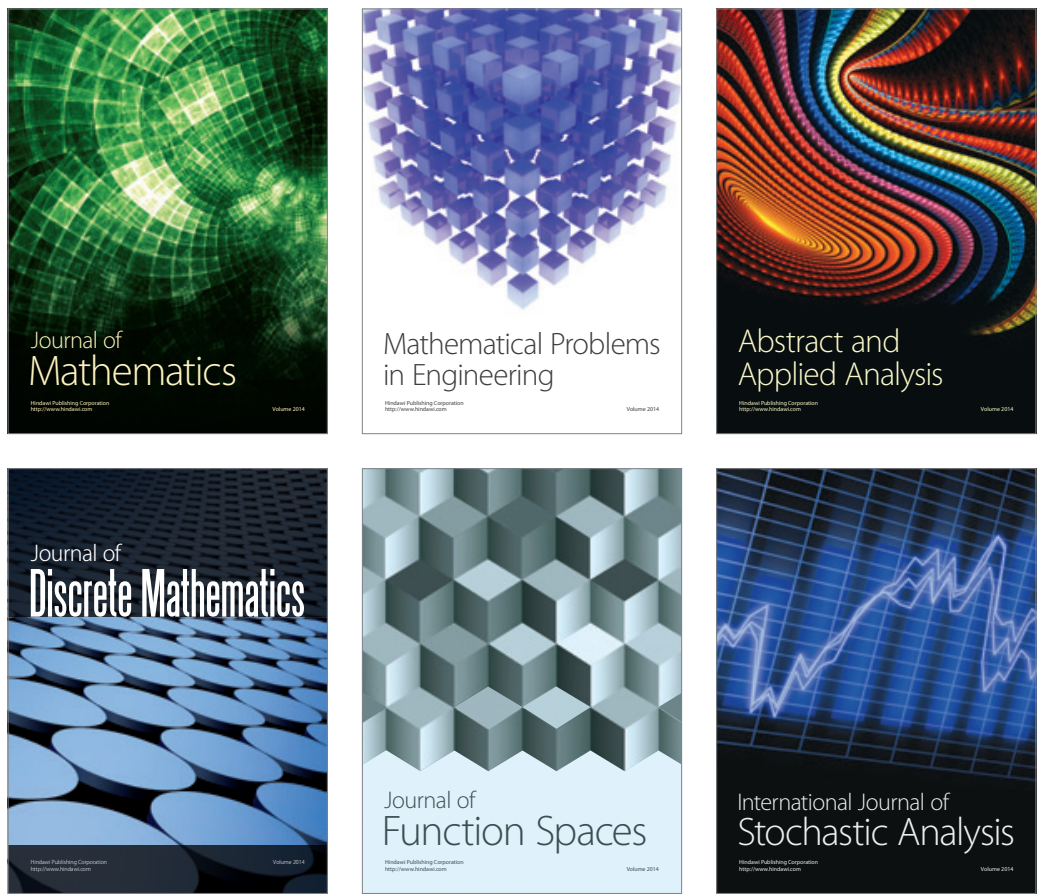

Journal of

Function Spaces

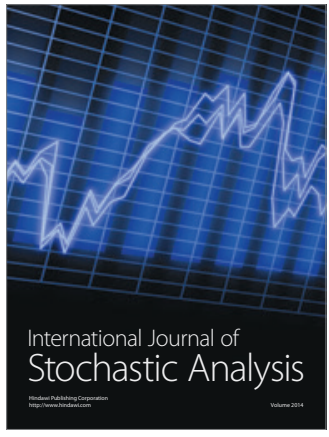

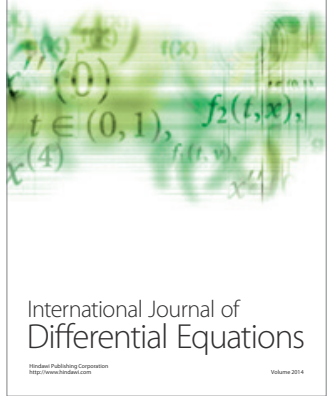
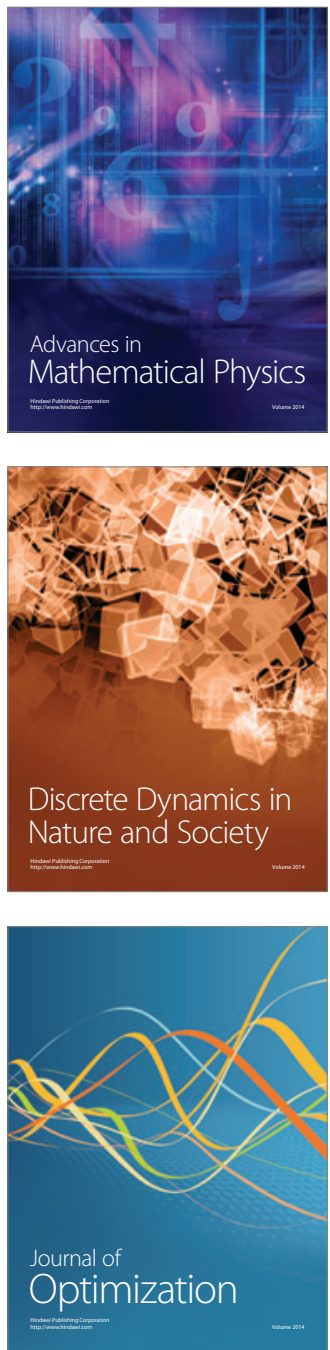